\section{AN ANNOTATED CHECKLIST OF ODONATA (INSECTA) OF KANHA TIGER RESERVE AND ADJOINING AREAS, CENTRAL INDIA}

\section{Pradeep K. Sahoo ${ }^{1}$, Sunit K. Das ${ }^{2}$ \& S.P. Parida ${ }^{3}$}

${ }^{1}$ Barunei Nodal U.P. School, At-Mutunia, PO-Bhandisahi, Via-Kakatpur, Puri, Odisha 752108 , India

${ }^{2}$ Wildlife Institute of India, Chandrabani, Dehradun, Uttarakhand 248001, India

${ }^{3}$ Regional Museum of Natural History, Acharya Vihar, Bhubaneswar, Odisha 751013, India

${ }^{1}$ pradeepgrasshopper@gmail.com, ${ }^{2}$ sunit.das219@gmail.com (corresponding author), ${ }^{3}$ paridasp@gmail.com

The study was carried out at the Kanha Tiger Reserve (KTR) India, which is a major conservation area. It is an internationally renowned protected area in India and located in the Maikal ranges at the eastern base of the triangular Satpura range in the central Indian highlands of Madhya Pradesh. Kanha is known for its exceptional natural beauty and its unique and diverse flora and fauna. The immense natural beauty of Kanha even inspired the famous author Rudyard Kipling to write his all time classic 'Jungle Book'. The area is well known for big-cats and harbors an average population of 60 (4575) Bengal Tigers Panthera tigris (Jhala et al. 2011) with their prey base and also supports more than 200 species of birds (Newton et al. 1986). But very little information on invertebrates like odonates is available. They are one of the least studied groups of insects though they are known as bio-indicators in the natural ecosystem (Watson et al. 1982). Joshi et al. 2004, studied the insect fauna of Kanha, but Odonata (Insecta) fauna of the reserve was primarily revealed in scientific literature such as Fraser (1933, 1934, 1936) and Tiple et al. (2010). The present study aimed at collecting baseline information on this magnificent group of insects and covered the Kanha Tiger Reserve including the core and buffer areas.

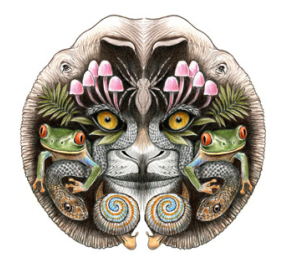

ISSN

Online 0974-7907 Print 0974-7893

OPEN ACCESS

\section{Study area}

The study area included the Supkhar range of the Kanha National Park which is $35 \mathrm{~km}$ from Mukki gate of Mukki range of the Kanha National Park. It is in the Balaghat District of Madhya Pradesh. It lies between $22^{\circ} 20^{\prime} \mathrm{N} \& 80^{\circ} 38^{\prime} \mathrm{E}$ and the reserve occupies an area of $940 \mathrm{~km}^{2}$. The Kanha Tiger Reserve includes the area of two sanctuaries namely Hallon and Banjar, of 250 and $300 \mathrm{~km}^{2}$ respectively, together with a surrounding buffer zone of $1,009 \mathrm{~km}^{2}$ and the neighboring $110 \mathrm{~km}^{2}$ Phen Sanctuary (Image 1).

Vegetation: The broad vegetation of Kanha Tiger Reserve is dry deciduous forest. The terrain is undulating. Champion \& Seth (1968) have identified the following forest types in Kanha: (i) moist peninsular sal forest (a - high level sal, b - low level sal, c - valley sal); (ii) southern tropical moist deciduous forest; (iii) southern tropical dry mixed deciduous forest. The floral diversity comprises 609 species and 10 varieties of angiosperms belonging to 386 genera and 104 families and 17 species of pteridophytes belonging to 11 genera and nine families (Dash 2010). The major tree species are Sal Shorea robusta, Saja Terminalia alata, Lendia Lagerstroemia parviflora, Dhawa Anogeissus latifolia, Tendu Diospyros melanoxylon, Palash Butea monosperma, Bija Pterocarpus marsupium, Mahua Madhuca indica, Aonla Emblica officianalis, Achar Buchanania lanzan. The

DOI: http://dx.doi.org/10.11609/JoTT.02822.476 | ZooBank: urn:Isid:zoobank.org:pub:1CECBE2A-29B5-472E-B72A-317C146FED7A

Editor: K.A. Subramanian, Zoological Survey of India, Kolkata, India

Date of publication: 26 January 2013 (online \& print)

Manuscript details: Ms \# 02822 | Received 06 June 2011 | Final received 17 July 2012 | Finally accepted 31 December 2012

Citation: Sahoo, P.K., S.K. Das \& S.P. Parida (2013). An annotated checklist of Odonata (Insecta) of Kanha Tiger Reserve and adjoining areas, central India. Journal of Threatened Taxa 5(1): 3559-3564; doi:10.11609/JoTT.o2822.476

Copyright: @ Sahoo et al. 2013. Creative Commons Attribution 3.0 Unported License. JoTT allows unrestricted use of this article in any medium, reproduction and distribution by providing adequate credit to the authors and the source of publication.

Funding: None.

Competing Interest: None.

Acknowledgements: The authors are grateful to Dr. K.A. Subramanian, Zoological Survey of India for his valuable suggestions and Dr. A.D. Tiple for valuable support. The first and second author thank to Mr. Lalthanpuia, Amol Kumbhar, Anil Dashahare, Ujjwal Sinha, Bidyut Bikash Barman, Madhura Davate and Neha Avasti of Wildlife Institute of India for the support during field data collection. We are grateful to Prof. Y.V. Jhala and Prof. Qamar Qureshi and other staff of Wildlife Institute of India for their valuable suggestions and guidance. We also want to acknowledge Dr. H.S. Negi (Field Director), Mr. H.S. Mohanta (Deputy Director), Dr. Rakesh Shukla (Research Officer) and other staff members of Kanha Tiger Reserve for their constant support and encouragement for writing this research article. 
major grass species include Eragostis sp., Andropogon sp., Cynodon sp., and Bambusa sp.

\section{Methods}

The study was carried out from January 2010 to December 2010. Odonates are most active during midday, (Subramanian 2005) therefore, direct search technique (Sutherland 1996) was used during this period (1000$1400 \mathrm{hr}$ ). Opportunistic sightings were also recorded. The identification of dragonflies and damselflies is based on Subramanian (2009). Photographs were taken with a Nikon P90 digital camera with double close up mode. Odonates are categorized into three groups based on the observations during the period of study. Accordingly, those species observed on $75-100 \%$ of the survey days were categorized as very common (A), 50-75\% as common (C), 25-50\% as occasional (OC) and below $25 \%$ as rare (R). We surveyed mainly the banks of major rivers such as Banjar and Hallon with surveys of perennial and seasonal streambeds of all types of forest habitats of the reserve. We also surveyed the major tals (lakes) such as Sharvan Tal, Phuta Tal, Sunder Tal which serve as prime habitats for odonates in and around the reserve (Image 1).

\section{Result and Discussion}

Madhya Pradesh provides a suitable habitat to 72 species of odonates. Mishra (2000) and Andrew et al. (2008) listed common 45 species of odonata from central India. Six species of odonates were previously recorded from Panchmarhi Biosphere Reserve, Madhya Pradesh (ZSI 2009). Chandra (2009) reported the distribution of eight species of odonates from Bandhavgarh Tiger Reserve. From Kanha 36 species of odonates were previously recorded (Tiple et al. 2010), with 34 genera. The present study records the distribution of 38 species of odonates in Kanha Tiger Reserve including seven families and 26 genera, where 12 species distribution are recorded for the first time. The family Libellulidae (21) is well represented in the present study followed by Coenagrionidae (8), Calopterygidae (2), Gomphidae (2), Lestidae (2) and Aeshnidae (3). In the case of zygopterans, Ischnura aurora (Brauer) was more abundant than the others. Among the collected libelluids, Orthretum sabina sabina (Drury) was the most abundant species. The family and species level classification follows Subramanian (2009). With the addition of these 12 newly recorded species, the existing checklist of Kanha is updated (Tiple et al. 2010) and the species richness

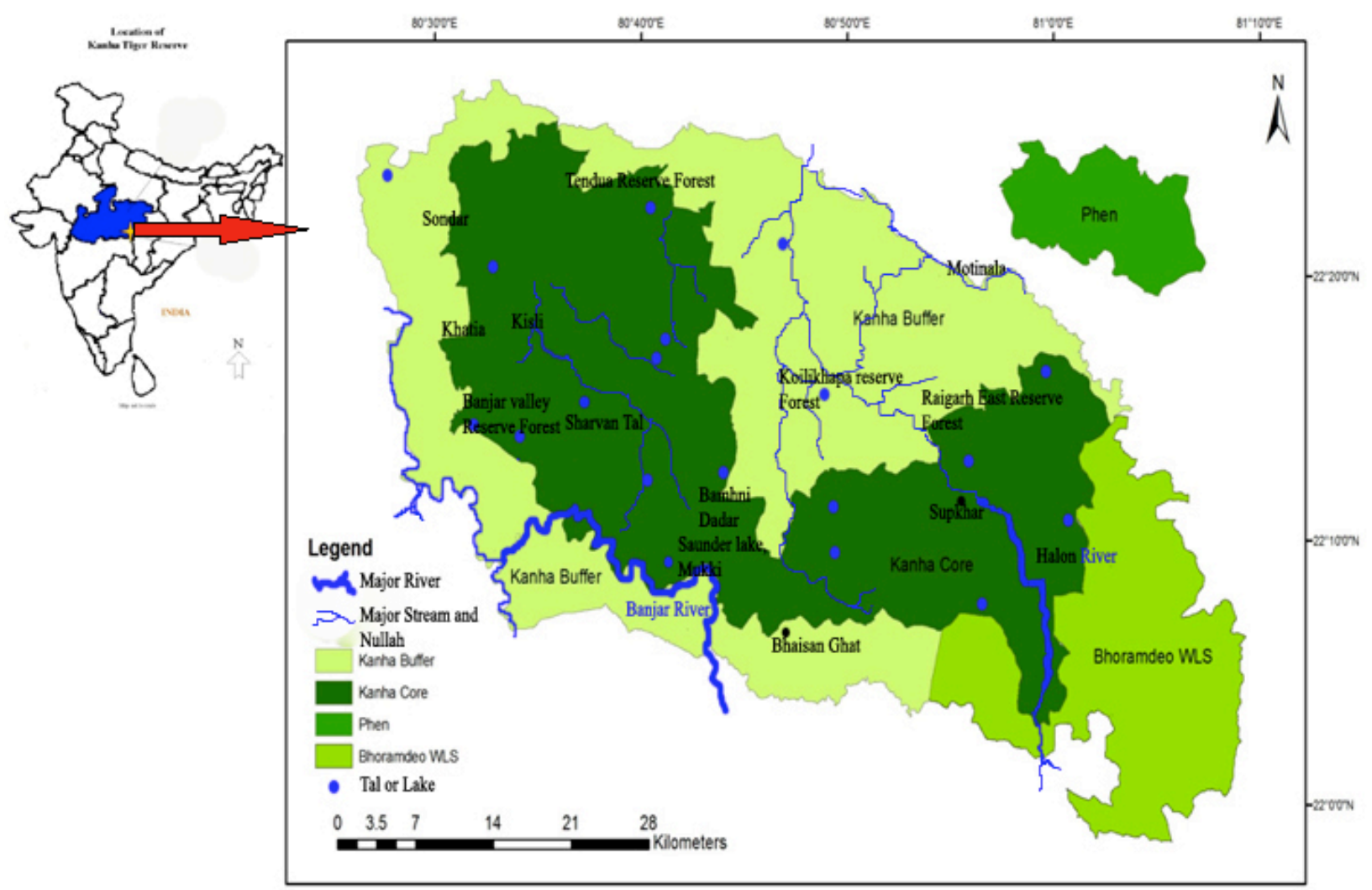

Image 1. Kanha Tiger Reserve with the water bodies surveyed for odonates 


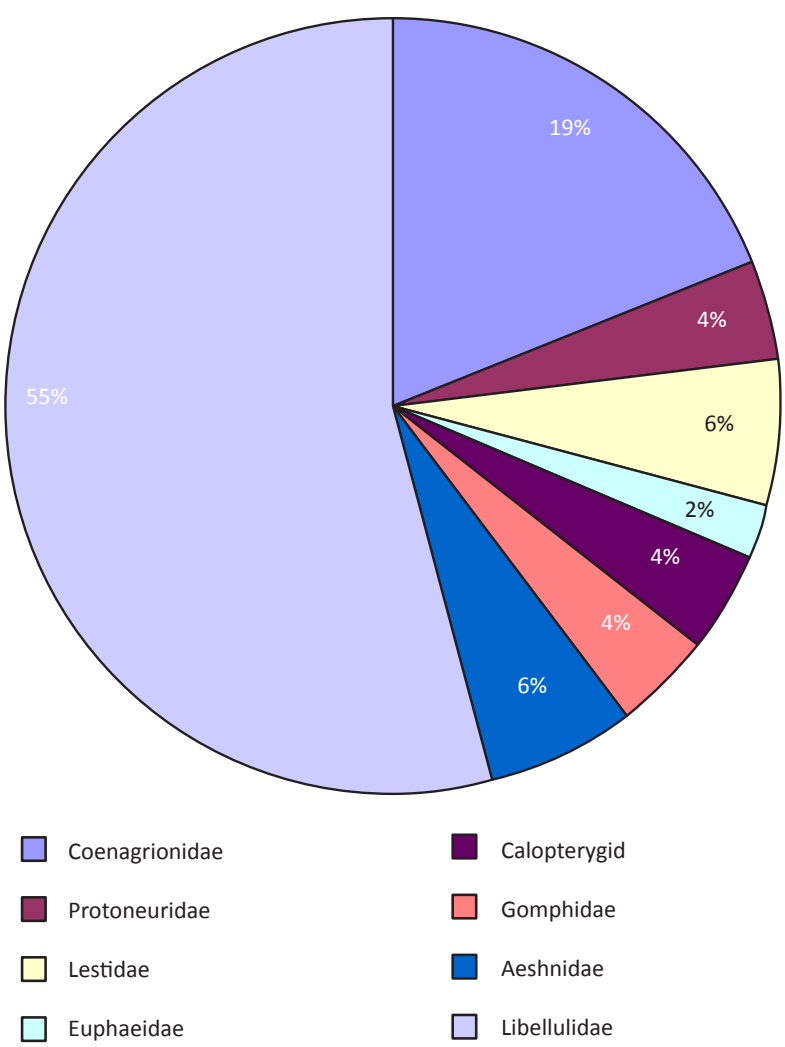

Figure 1. Percentage of species from each family

in the reserve has increased to 48 species, belonging to eight families (Table 1; Fig. 1). The checklist provides the status for only 38 species as 10 species reported in earlier studies were not recorded during the present study (Table 1). On the basis of direct sightings we found that of 38 species, seven are abundant, 25 common, five occasional and one rare as it was sighted only once during the field study.

Odonates are one of most significant groups of insects with their impact on the ecosystem as bio-indicators, predators and as prey for many living organisms from birds to other arthropods like spiders. The prey of the adults consists mostly of insects harmful to crops, orchards and forests, thus they have a regulatory impact on agro-forestry. Public attentiveness is requisite to conserve these odonates and their habitats. The study reflects the baseline information on these beautiful groups of insects, but is limited in explanation of their use of different habitat types in the central Indian landscape. Therefore, an extensive odonatological survey needs to be carried out to explore the rich diversity of these elegant insects with their richness in different forest vegetation.
Table 1. Annotated checklist of odonates of Kanha Tiger Reserve, central India

\begin{tabular}{|c|c|c|c|c|}
\hline & Family and species * & $\begin{array}{l}\text { Tiple } \\
\text { et al. } \\
2010\end{array}$ & $\begin{array}{l}\text { Present } \\
\text { Study }\end{array}$ & Status \\
\hline & Coenagrionidae & & & \\
\hline 1 & $\begin{array}{l}\text { Agriocnemis pygmea (Rambur, } \\
\text { 1842) }\end{array}$ & r & $r$ & A \\
\hline 2 & $\begin{array}{l}\text { Ceriagrion coromandelianum } \\
\text { (Fabricius, 1798) }\end{array}$ & $r$ & $r$ & A \\
\hline 3 & $\begin{array}{l}\text { Ceriagrion olivaceum Laidlaw, } \\
1914\end{array}$ & $\mathrm{nr}$ & r & C \\
\hline 4 & $\begin{array}{l}\text { Ischnura aurora (Brauer, 1865) } \\
\text { (Image 2) }\end{array}$ & $r$ & $r$ & A \\
\hline 5 & $\begin{array}{l}\text { Ischnura senegalensis (Rambur, } \\
\text { 1842) (Image 3) }\end{array}$ & r & $r$ & C \\
\hline 6 & $\begin{array}{l}\text { Pseudagrion microcephalum } \\
\text { (Rambur, 1842) (Image 4) }\end{array}$ & $r$ & $r$ & C \\
\hline 7 & $\begin{array}{l}\text { Pseudagrion rubriceps Selys, } \\
1876\end{array}$ & $r$ & $r$ & C \\
\hline 8 & $\begin{array}{l}\text { Pseudagrion decorum Rambur, } \\
1842\end{array}$ & $r$ & $r$ & C \\
\hline \multirow[t]{2}{*}{9} & $\begin{array}{l}\text { Rhodischnura nursei Morton, } \\
1907\end{array}$ & $r$ & $r$ & A \\
\hline & Protoneuridae & & & \\
\hline 10 & $\begin{array}{l}\text { Disparoneura quadrimaculata } \\
\text { (Rambur, 1842) }\end{array}$ & $r$ & $r$ & $\mathrm{R}$ \\
\hline \multirow[t]{2}{*}{11} & $\begin{array}{l}\text { Prodasineura verticalis (Selys, } \\
1860 \text { ) }\end{array}$ & $r$ & $r$ & A \\
\hline & Lestidae & & & \\
\hline 12 & Lestes viridulus Rambur, 1842 & $\mathrm{nr}$ & $r$ & C \\
\hline 13 & $\begin{array}{l}\text { Lestes elatus Hagen in Selys, } \\
1862\end{array}$ & $\mathrm{nr}$ & $r$ & C \\
\hline \multirow[t]{2}{*}{14} & Lestes umbrinus Selys, 1891 & $r$ & $r$ & $\mathrm{R}$ \\
\hline & Euphaeidae & & & \\
\hline \multirow[t]{2}{*}{15} & Dysphaea ethela Fraser, 1924 & r & $r$ & $\mathrm{R}$ \\
\hline & Calopterygidae & & & \\
\hline 16 & $\begin{array}{l}\text { Neurobasis chinensis (Linnaeus, } \\
1758 \text { ) }\end{array}$ & $\mathrm{nr}$ & $r$ & C \\
\hline \multirow[t]{2}{*}{17} & Vestalis apicalis Selys, 1873 & $\mathrm{nr}$ & $r$ & C \\
\hline & Gomphidae & & & \\
\hline 18 & $\begin{array}{l}\text { Ictinogomphus rapax Rambur, } \\
1842 \text { (Image 5) }\end{array}$ & $r$ & r & C \\
\hline \multirow[t]{2}{*}{19} & $\begin{array}{l}\text { Paragomphus lineatus (Selys, } \\
\text { 1850) }\end{array}$ & $r$ & r & C \\
\hline & Aeshnidae & & & \\
\hline 20 & $\begin{array}{l}\text { Anaciaeschna jaspidea } \\
\text { (Burmeister, 1839) }\end{array}$ & $\mathrm{nr}$ & $r$ & OC \\
\hline 21 & $\begin{array}{l}\text { Anax guttatus (Burmeister, } \\
\text { 1839) }\end{array}$ & $r$ & $r$ & OC \\
\hline \multirow[t]{2}{*}{22} & $\begin{array}{l}\text { Anax immaculifrons Rambur, } \\
1842\end{array}$ & $r$ & $r$ & OC \\
\hline & Libellulidae & & & \\
\hline 23 & $\begin{array}{l}\text { Acisoma panorpoides Rambur, } \\
1842\end{array}$ & $\mathrm{nr}$ & $r$ & C \\
\hline 24 & $\begin{array}{l}\text { Aethriamanta brevipennis } \\
\text { (Rambur, 1842) }\end{array}$ & $\mathrm{nr}$ & $r$ & C \\
\hline
\end{tabular}




\begin{tabular}{|c|c|c|c|c|}
\hline & Family and species * & $\begin{array}{l}\text { Tiple } \\
\text { et al. } \\
2010\end{array}$ & $\begin{array}{l}\text { Present } \\
\text { Study }\end{array}$ & Status \\
\hline 25 & $\begin{array}{l}\text { Brachydiplax farinosa Krüger, } \\
1902\end{array}$ & $r$ & $r$ & $\mathrm{R}$ \\
\hline 26 & $\begin{array}{l}\text { Brachythemis contaminata } \\
\text { (Fabricius, 1793) }\end{array}$ & $r$ & r & C \\
\hline 27 & $\begin{array}{l}\text { Bradinopyga geminata } \\
\text { (Rambur, 1842) }\end{array}$ & $r$ & r & C \\
\hline 28 & $\begin{array}{l}\text { Crocothemis servilia (Drury, } \\
\text { 1770) }\end{array}$ & $r$ & r & C \\
\hline 29 & $\begin{array}{l}\text { Diplacodes trivialis (Rambur, } \\
\text { 1842) }\end{array}$ & $r$ & $r$ & A \\
\hline 30 & $\begin{array}{l}\text { Diplacodes nebulosa (Frbricius, } \\
\text { 1793) }\end{array}$ & r & $r$ & A \\
\hline 31 & $\begin{array}{l}\text { Lathrecista asiatica (Fabricius, } \\
\text { 1798) }\end{array}$ & $r$ & r & A \\
\hline 32 & $\begin{array}{l}\text { Neurothemis fulvia (Drury, } \\
1773 \text { ) }\end{array}$ & $r$ & r & A \\
\hline 33 & Neurothemis tullia (Drury, 1773) & $\mathrm{nr}$ & r & C \\
\hline 34 & $\begin{array}{l}\text { Neurothemis intermedia } \\
\text { (Rambur, 1842) }\end{array}$ & $r$ & $r$ & A \\
\hline 35 & $\begin{array}{l}\text { Orthetrum pruinosum (Rambur, } \\
\text { 1842) (Image 6) }\end{array}$ & r & $r$ & C \\
\hline 36 & Orthetrum sabina (Drury, 1770) & r & r & A \\
\hline 37 & $\begin{array}{l}\text { Orthetrum triangulare (Selys, } \\
\text { 1878) }\end{array}$ & $\mathrm{nr}$ & $r$ & $\mathrm{OC}$ \\
\hline 38 & $\begin{array}{l}\text { Pantala flavescens (Fabricius, } \\
\text { 1798) }\end{array}$ & r & r & A \\
\hline 39 & $\begin{array}{l}\text { Potamarcha congener (Rambur, } \\
\text { 1842) (Image 7) }\end{array}$ & $r$ & r & C \\
\hline 40 & $\begin{array}{l}\text { Rhodothemis rufa (Rambur, } \\
\text { 1842) }\end{array}$ & $\mathrm{nr}$ & $\mathrm{r}$ & C \\
\hline 41 & $\begin{array}{l}\text { Rhyothemis variegate Linnaeus, } \\
1763\end{array}$ & $\mathrm{nr}$ & $r$ & C \\
\hline 42 & $\begin{array}{l}\text { Tetrathemis platyptera Selys, } \\
1878\end{array}$ & r & r & LC \\
\hline 43 & $\begin{array}{l}\text { Tholymis tillarga (Fabricius, } \\
\text { 1798) }\end{array}$ & r & r & C \\
\hline 44 & Tramea basilaris Kirby, 1889 & r & $\mathrm{r}$ & C \\
\hline 45 & $\begin{array}{l}\text { Trithemis aurora (Burmeister, } \\
\text { 1839) }\end{array}$ & $r$ & r & C \\
\hline 46 & $\begin{array}{l}\text { Trithemis festiva (Rambur, } \\
\text { 1842) }\end{array}$ & $r$ & $r$ & C \\
\hline 47 & Trithemis kirbyi Selys, 1891 & r & r & $\mathrm{OC}$ \\
\hline 48 & $\begin{array}{l}\text { Trithemis pallidinervis Selys, } \\
1889\end{array}$ & $r$ & $r$ & C \\
\hline
\end{tabular}

$\mathrm{r}$ - recorded; $\mathrm{nr}$ - not recorded; A - Abundant, C - Common, dd - data deficit, LC Lease concern; OC - Occasional, R - Rare; * - Name as in Subramanian 2009.

\section{REFERENCE}

Andrew, R.J., K.A. Subramaniam \& A.D. Tiple (2008). Common Odonates of Central India. E-book for "The $18^{\text {th }}$ International Symposium of Odonatology", Hislop College, Nagpur, India, 55pp. Champion, H.G. \& S.K. Seth (1968). A Revised Survey of the Forest Types of India. The Manager of Publications, Government of India, New Delhi, 404pp.

Chandra, K. (2009). An Overview. Zoological Survey of Fauna of Bandhabgarh Tiger Reserve. Conservation Area Series 40: 1-24.

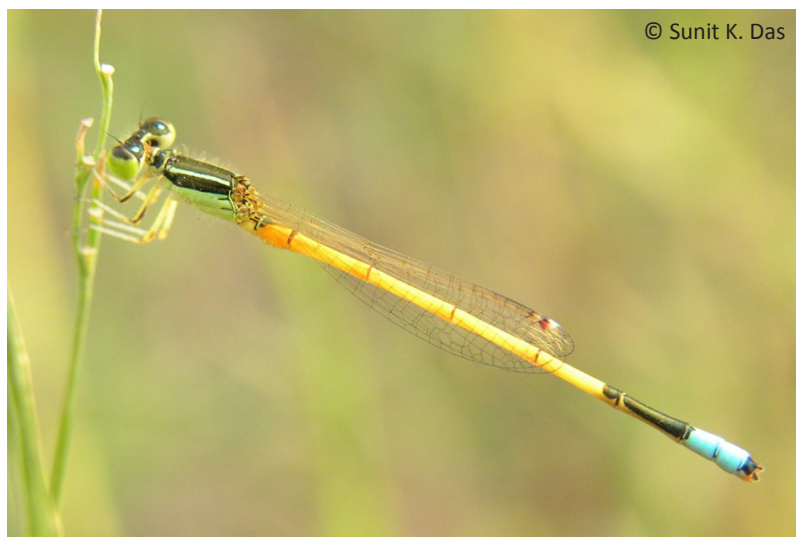

Image 2. Ischnura aurora

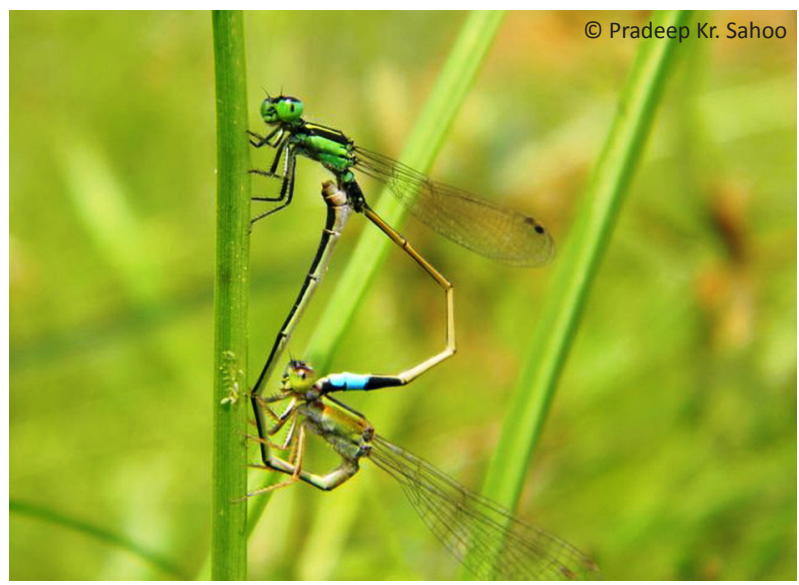

Image 3. Ischnura senegalensis

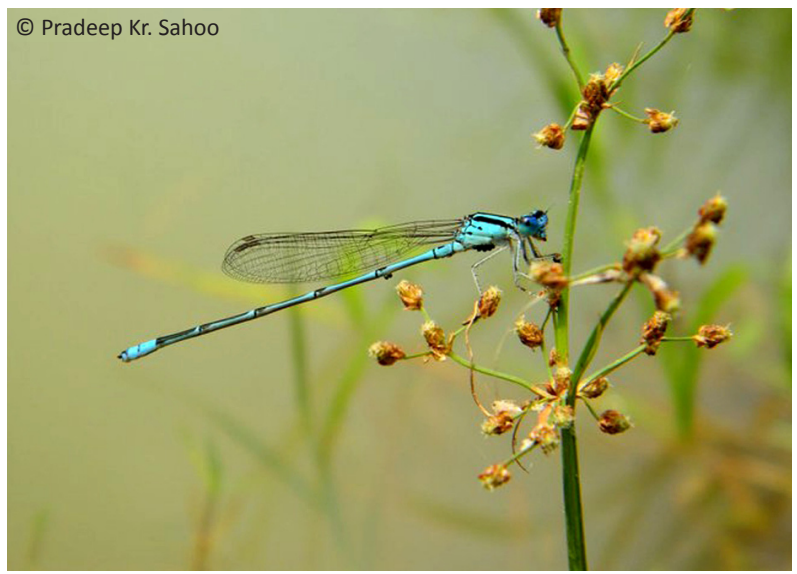

Image 4. Pseudagrion microcephalum 


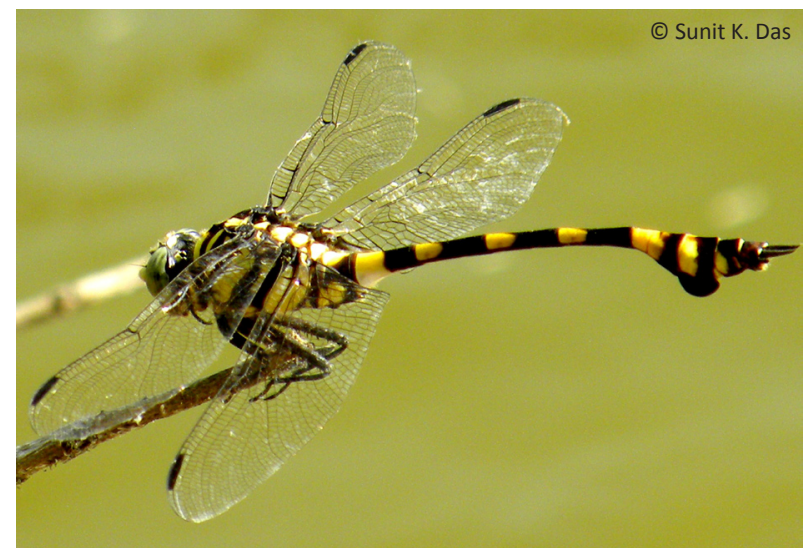

Image 5. Ictinogomphus rapax

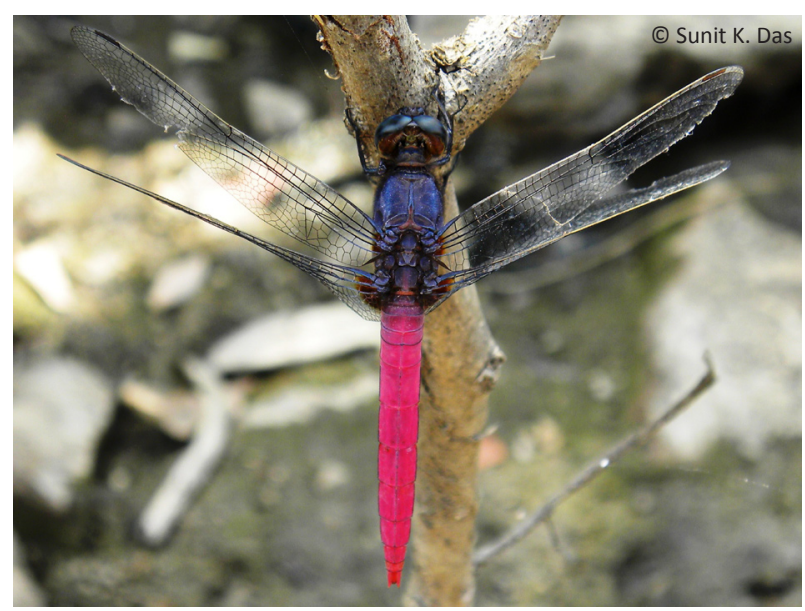

Image 6. Orthetrum pruinosum

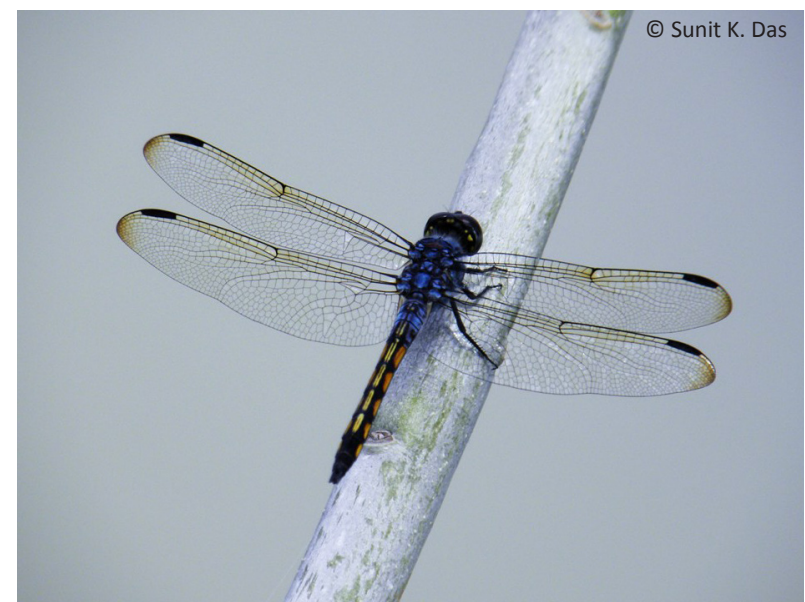

Image 7. Potamarcha congener
Fraser, F.C. (1933). The Fauna of British-India including Ceylon and Burma, Odonata-Vol. I. Taylor and Francis Ltd., London, 436pp.

Fraser, F.C. (1934). The Fauna of British-India including Ceylon and Burma, Odonata-Vol. II. Taylor and Francis Ltd., London, 442pp.

Fraser, F.C. (1936). The Fauna of British-India including Ceylon and Burma, Odonata. Vol. -III. Taylor and Francis Ltd., London, 448pp.

Jhala, Y.V., Q. Qureshi, R. Gopal \& P.R. Sinha (eds.) (2011). Status of the Tigers, Co-predators, and Prey in India. National Tiger Conservation Authority, Govt. of India, New Delhi, and Wildlife Institute of India, Dehradun, 302pp.

Joshi, K.C., N. Kulkarni, N. Roychoudhury, S. Chandra \& S. Barve (2004). A study of insects from Kanha National Park. Journal of Tropical Forestry 20(III \& IV): 58-74.

Mitra, T.R. (2002). Geographical Distribution of Odonata (Insecta) of Eastern India. Memories of the Zoological Survey of India 19(1): 171.

Mishra, S.K. (2000). Fauna of Madhya Pradesh (Odonata: Insecta). Zoological Survey of India, Kolkata.

Newton, P.N., S. Breeden \& G.J. Norman (1986). The Birds of Kanha Tiger Reserve, Madhya Pradesh, India, Journal of the Bombay Natural History Society 83: 477-498.

Subramanian, K.A. (2005). Dragonflies and Damselflies of Peninsular India - A Field Guide. Vigyan Prasar, India Offset Press, New Delhi, $35 \mathrm{pp}$.

Subramanian, K.A. (2009). Dragonflies of India - A Field Guide. Vigyan Prasar, Department of Science and Technology, Govt. of India, $168 p p$.

Sutherland, W.J. (1996). Ecological Census Techniques. University Press, Cambridge, 200pp.

Tiple, A.D., N. Kulkarni \& K.C. Joshi (2010). Diversity of Odonata in Kanha National Park, Madhya Pradesh, India. Indian Journal of Forestry 34(3): 329-332.

Watson, J.A.L., A.H. Arthington \& D.L. Conrick (1982). Effect of sewage effluent ondragonflies (Odonata) of Bulimba Creek, Brisbane. Australian Journal of Marine and Freshwater Research 33: 517-28.

ZSI (2009). Fauna of Panchmarhi Biosphere Reserve: Madhya Pradesh. Conservation Area Series - 39, Zoological Survey of India, 380pp+20colour pls. 
Appendix 1. Presence or absence of every species in study area. $\mathbf{V}$ - Presence; $\mathbf{X}$ - Absence

\begin{tabular}{|c|c|c|c|c|c|c|c|}
\hline \multirow[t]{2}{*}{ Sno } & \multirow[t]{2}{*}{ Family and species } & \multicolumn{6}{|c|}{ Ranges of Kanha National Park and adjoining areas where study carried out } \\
\hline & & Kanha & Kisli & Mukki & Supkhar & Sarrhi & Bhaisan Ghat \\
\hline & \multicolumn{7}{|l|}{ Coenagrionidae } \\
\hline 1 & Agriocnemis pygmea (Rambur, 1842) & $\checkmark$ & $v$ & $\checkmark$ & $v$ & $\checkmark$ & $\sqrt{ }$ \\
\hline 2 & Ceriagrion coromandelianum (Fabricius, 1798) & $\sqrt{ }$ & $\mathrm{v}$ & $\checkmark$ & $\checkmark$ & $\checkmark$ & $\mathrm{v}$ \\
\hline 3 & Ceriagrion olivaceum Laidlaw, 1914 & $\mathrm{~V}$ & $\mathrm{v}$ & $\mathrm{v}$ & $\mathrm{v}$ & $\mathrm{v}$ & $\mathrm{v}$ \\
\hline 4 & Ischnura aurora (Brauer, 1865) & $\mathrm{v}$ & $\mathrm{v}$ & $\mathrm{v}$ & 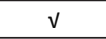 & $\mathrm{v}$ & $\mathrm{v}$ \\
\hline 5 & Ischnura senegalensis (Rambur, 1842) & $\mathrm{v}$ & $\mathrm{v}$ & $\mathrm{v}$ & $\mathrm{v}$ & $\mathrm{v}$ & $x$ \\
\hline 6 & Pseudagrion microcephalum (Rambur, 1842) & 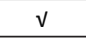 & $v$ & $v$ & $v$ & $\checkmark$ & $\mathrm{x}$ \\
\hline 7 & Pseudagrion rubriceps Selys, 1876 & $\mathrm{v}$ & $\mathrm{v}$ & 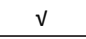 & $v$ & 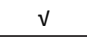 & $\mathrm{x}$ \\
\hline 8 & Pseudagrion decorum Rambur, 1842 & $\mathrm{v}$ & $\mathrm{v}$ & $\checkmark$ & $\checkmark$ & $\checkmark$ & $x$ \\
\hline \multirow[t]{2}{*}{9} & Rhodischnura nursei Morton, 1907 & 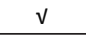 & $x$ & $v$ & $x$ & $x$ & $v$ \\
\hline & \multicolumn{7}{|l|}{ Protoneuridae } \\
\hline 10 & Disparoneura quadrimaculata (Rambur, 1842) & 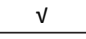 & $v$ & $v$ & $v$ & $\mathrm{x}$ & $x$ \\
\hline \multirow[t]{2}{*}{11} & Prodasineura verticalis (Selys, 1860) & $\checkmark$ & 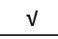 & $v$ & $\checkmark$ & $x$ & $\mathrm{x}$ \\
\hline & \multicolumn{7}{|l|}{ Lestidae } \\
\hline 12 & Lestes viridulus Rambur, 1842 & $\checkmark$ & $v$ & 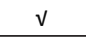 & $v$ & $\mathrm{x}$ & $\mathrm{x}$ \\
\hline 13 & Lestes elatus Hagen in Selys, 1862 & $\checkmark$ & $\mathrm{v}$ & $\checkmark$ & $\checkmark$ & $x$ & $\mathrm{x}$ \\
\hline \multirow[t]{2}{*}{14} & Lestes umbrinus Selys, 1891 & $v$ & $v$ & $v$ & $v$ & $x$ & $\mathrm{x}$ \\
\hline & \multicolumn{7}{|l|}{ Euphaeidae } \\
\hline \multirow[t]{2}{*}{15} & Dysphaea ethela Fraser, 1924 & $\checkmark$ & $\mathrm{v}$ & $\checkmark$ & $\mathrm{v}$ & $x$ & $x$ \\
\hline & \multicolumn{7}{|l|}{ Calopterygidae } \\
\hline 16 & Neurobasis chinensis (Linnaeus, 1758) & $\sqrt{ }$ & $\mathrm{v}$ & $\mathrm{v}$ & $\mathrm{v}$ & $\mathrm{x}$ & $\mathrm{x}$ \\
\hline \multirow[t]{2}{*}{17} & Vestalis apicalis Selys, 1873 & $\checkmark$ & $v$ & $\checkmark$ & $\checkmark$ & $x$ & $x$ \\
\hline & \multicolumn{7}{|l|}{ Gomphidae } \\
\hline 18 & Ictinogomphus rapax Rambur, 1842 & 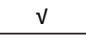 & $v$ & $v$ & $v$ & $x$ & $x$ \\
\hline \multirow[t]{2}{*}{19} & Paragomphus lineatus (Selys, 1850) & $v$ & $v$ & $v$ & $\checkmark$ & $x$ & $x$ \\
\hline & \multicolumn{7}{|l|}{ Aeshnidae } \\
\hline 20 & Anaciaeschna jaspidea (Burmeister, 1839) & 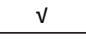 & & $v$ & $v$ & $x$ & $x$ \\
\hline 21 & Anax guttatus (Burmeister, 1839) & $\checkmark$ & & $\checkmark$ & $v$ & $x$ & $x$ \\
\hline 22 & Anax immaculifrons Rambur, 1842 & $\checkmark$ & & $\checkmark$ & $\checkmark$ & $x$ & $\mathrm{x}$ \\
\hline & Libellulidae & & & & & & \\
\hline 23 & Acisoma panorpoides Rambur, 1842 & $v$ & $v$ & $v$ & $\checkmark$ & $v$ & $\mathrm{x}$ \\
\hline 24 & Aethriamanta brevipennis (Rambur, 1842) & $\checkmark$ & $v$ & $v$ & $v$ & $\checkmark$ & $\mathrm{x}$ \\
\hline 25 & Brachydiplax farinosa Krüger, 1902 & $x$ & $x$ & $x$ & $v$ & $x$ & $x$ \\
\hline 26 & Brachythemis contaminata (Fabricius, 1793) & $\mathrm{v}$ & $\mathrm{v}$ & $\mathrm{v}$ & $\mathrm{v}$ & $\mathrm{v}$ & $\checkmark$ \\
\hline 27 & Bradinopyga geminata (Rambur, 1842) & $\mathrm{v}$ & $\mathrm{v}$ & 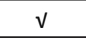 & $v$ & $\mathrm{v}$ & $\checkmark$ \\
\hline 28 & Crocothemis servilia (Drury, 1770) & 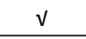 & $\mathrm{v}$ & $\mathrm{v}$ & $v$ & $\checkmark$ & $\checkmark$ \\
\hline 29 & Diplacodes trivialis (Rambur, 1842) & $\checkmark$ & $v$ & $v$ & $\checkmark$ & $\checkmark$ & $v$ \\
\hline 30 & Diplacodes nebulosa (Frbricius, 1793) & $v$ & $v$ & $v$ & $v$ & $v$ & $v$ \\
\hline 31 & Lathrecista asiatica (Fabricius, 1798) & 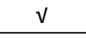 & $v$ & $v$ & $v$ & $v$ & 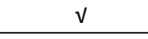 \\
\hline 32 & Neurothemis fulvia (Drury, 1773) & $v$ & $v$ & $v$ & $v$ & $v$ & $\mathrm{v}$ \\
\hline 33 & Neurothemis tullia (Drury, 1773) & 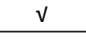 & $\mathrm{v}$ & v & $v$ & 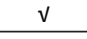 & $\mathrm{v}$ \\
\hline 34 & Neurothemis intermedia (Rambur, 1842) & $v$ & $v$ & $v$ & $v$ & $v$ & $v$ \\
\hline 35 & Orthetrum pruinosum (Rambur, 1842) (Image 6) & $\checkmark$ & $\mathrm{v}$ & 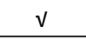 & 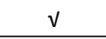 & $\checkmark$ & $\mathrm{v}$ \\
\hline 36 & Orthetrum sabina (Drury, 1770) & $\checkmark$ & $\mathrm{v}$ & $\checkmark$ & $\checkmark$ & 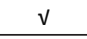 & $\mathrm{v}$ \\
\hline 37 & Orthetrum triangulare (Selys, 1878) & 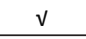 & $\mathrm{v}$ & $\checkmark$ & $\checkmark$ & $\checkmark$ & 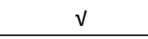 \\
\hline 38 & Pantala flavescens (Fabricius, 1798) & 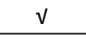 & $\mathrm{v}$ & $v$ & $v$ & 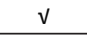 & $v$ \\
\hline 39 & Potamarcha congener (Rambur, 1842) (Image 7) & $v$ & $v$ & $v$ & $v$ & $v$ & $v$ \\
\hline 40 & Rhodothemis rufa (Rambur, 1842) & $\mathrm{v}$ & $\mathrm{v}$ & 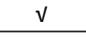 & $v$ & 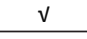 & $\mathrm{v}$ \\
\hline 41 & Rhyothemis variegate Linnaeus, 1763 & $\checkmark$ & $\mathrm{v}$ & $\mathrm{v}$ & $\mathrm{v}$ & $\mathrm{v}$ & $\mathrm{v}$ \\
\hline 42 & Tetrathemis platyptera Selys, 1878 & $\checkmark$ & $\mathrm{v}$ & $\mathrm{v}$ & $v$ & 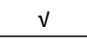 & $\mathrm{v}$ \\
\hline 43 & Tholymis tillarga (Fabricius,1798) & $\checkmark$ & $\mathrm{v}$ & $\checkmark$ & $\checkmark$ & $\checkmark$ & $\mathrm{V}$ \\
\hline 44 & Tramea basilaris Kirby, 1889 & $v$ & 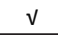 & $v$ & $v$ & $v$ & $\mathrm{v}$ \\
\hline 45 & Trithemis aurora (Burmeister, 1839) & $v$ & $v$ & $v$ & $v$ & $v$ & $\mathrm{~V}$ \\
\hline 46 & Trithemis festiva (Rambur, 1842) & $v$ & $\mathrm{v}$ & $v$ & $v$ & $v$ & $\mathrm{v}$ \\
\hline 47 & Trithemis kirbyi Selys, 1891 & $v$ & $v$ & $v$ & $v$ & $v$ & $\mathrm{v}$ \\
\hline 48 & Trithemis pallidinervis Selys, 1889 & $\checkmark$ & $v$ & $\checkmark$ & $v$ & $x$ & $x$ \\
\hline
\end{tabular}

